Volume 18, No 4 International Journal of Radiation Research, October 2020

\title{
Geometric distortion evaluation of magnetic resonance images by a new large field of view phantom for magnetic resonance based radiotherapy purposes
}

\author{
A. Rostami ${ }^{1}$,Sh. Naseri ${ }^{*}$, M. Momennezhad3, H. Zare², K. Anvari4, \\ H.R. Sayah Badkhor5 \\ ${ }^{1}$ Department of Medical Physics and Radiological Sciences, Faculty of Paramedical Sciences, Sabzevar University of \\ Medical Sciences, Sabzevar, Iran \\ ${ }^{2}$ Medical Physics Research Center, Mashhad University of Medical Sciences, Mashhad, Iran \\ ${ }^{3}$ Nuclear Medicine Research Center, Mashhad University of Medical Sciences, Mashhad, Iran \\ ${ }^{4}$ Department of Radiotherapy Oncology and Cancer Research Center, Mashhad University of Medical Sciences, \\ Mashhad, Iran \\ ${ }^{5}$ Kowsar MRI Center, Emam Reza Hospital, Mashhad, Iran
}

\begin{abstract}
- Original article

*Corresponding authors:

Shahrokh Naseri, Ph.D.,

E-mail: NaseriSH@mums.ac.ir

Revised: August 2019

Accepted: October 2019

Int. J. Radiat. Res., October 2020; 18(4): 733-742

DOI: 10.18869 /acadpub.ijrr.18.4.733

Background: The magnetic resonance imaging (MRI)-based radiotherapy planning method have been considered in recent years because of the advantages of MRI and the problems of planning with two images modality. The first step in MRI-based radiotherapy is to evaluate magnetic resonance (MR) images geometric distortion. Therefore, the present study aimed to evaluate system related geometric distortion by a new large field of view phantom. Materials and Methods: A homemade phantom with Perspex sheets and plastic pipes containing water was built for evaluating MR images distortion. The phantom size is $48 \times 48 \times 37 \mathrm{~cm}^{3}$ and includes 325 water pipes. The study evaluated four different protocols from a $60 \mathrm{~cm}$ bore MAGNETOM ${ }^{\circledR}$ Symphony Syngo $1.5 \mathrm{~T}$ (Siemens). Results: It was found that the amount of distortion for all protocols is under $2 \mathrm{~mm}$ for the radial distances less than 10 $\mathrm{cm}$ (field of view $(\mathrm{FOV})=20 \mathrm{~cm}$ ), but distortion increased in radial distances greater than $10 \mathrm{~cm}$, and reached about $5 \mathrm{~cm}$ for radial distances greater than $25 \mathrm{~cm}$. Conclusion: Geometric distortion of each MR scanner has been shown to be dependent on the radio frequency (RF) sequence pulse (Spin echo or Gradient echo) and image parameters (echo time (TE), repetition time (TR), and receiver band-width)). The geometric distortion could be ignored for the FOV $<20 \mathrm{~cm}$ (for the head region), and must be evaluated and corrected for more FOVs before the MR only radiotherapy.
\end{abstract}

Keywords: Geometric distortion, phantom, computed tomography, magnetic resonance imaging, treatment planning radiotherapy.

\section{INTRODUCTION}

The gold standard of imaging modality for radiotherapy treatment planning is computed tomography (CT) because of the information it provides about electron density for dosimetry calculation, geometric accuracy, and generation of digitally reconstructed radiograph (DRR) for patient positioning set up (1). However, a major problem of the CT images is the low soft tissue contrast, which increases the probability of error in contouring stage especially for solid tumors in soft tissue of the brain, head, neck, and pelvic regions ${ }^{2}$. MR images have the superior 
soft tissue contrast compared to CT images beside of multi-planar imaging and functional and physiological imaging that can be helpful for accurate diagnosis and treatment (3-5).

Studies showed that for treatment planning purpose in common clinical applications, the fusion of CT and MR images is used in the contouring stage that have several uncertainties. On the other hand, the use of two imaging modalities increases workload and associated costs. Thus, the MR only radiotherapy has been studied in recent years due to the advantages of MR imaging and problems of planning with two images modality. However, geometric distortion of MR images is a major problem in radiotherapy applications (6).

In the MR imaging for routine diagnostic examinations, geometric distortion has little effect on the results, and the two-dimensional (2D) or three-dimensional (3D) distortion correction programs presented by each vendor are adequate. However, the amount of geometric distortion can be a serious problem in certain MR images applications such as MR-based radiotherapy, MR-based radiosurgery, and MR-based surgery where high geometric accuracy is required (7).

Several studies investigated geometric distortion of MR images and reported that distortion magnitudes could range between sub-mms and $>2 \mathrm{~cm}$. Put differently, if there is more than $1 \mathrm{~cm}$ error in the patient contour for a single $6 \mathrm{MV}$ beam, it will create more than 3\% error in dose calculation (8-10). Therefore, it is necessary to carefully evaluate the geometric accuracy when commissioning each MR simulator. In addition, spatial resolution accuracy should be less than $2 \mathrm{~mm}$ so that it can be acceptable for treatment planning (11).

Geometric distortions of MR images can be divided into two categories. 1 . The patient and system related distortion, both of which are unfavorable for MR planning; thus, they should be characterized and minimized as much as possible (12-14). 2. The object or patient related distortions that are originated from susceptibility, and chemical shift variations in the patient can be reduced by choosing suitable image parameters such as the use of a wider receiver bandwidth (at least $400 \mathrm{~Hz} /$ pixel) and fast $\mathrm{SE}$ sequences on fat saturation techniques (11, 15-17).

Studies have shown that inhomogeneities in the main magnetic field and gradient nonlinearity's generate the system-related distortions. Magnetic inhomogeneities are limited in modern MR scanners because of the shimming coils used. However, it has been expected that geometric distortion is small due to the static field inhomogeneity (18). In contrast, strong gradients with restricted length of the gradient coils, as well as the fewer turns used have increased the problems with gradient nonlinearity. For this reason, gradient nonlinearity in new fast systems is one of the major factors of geometric distortion of MR images (7).

Phantom-based techniques can provide accurate measurement of system related distortion (19). According to the results, the amount of image distortion is variable, and it is dependent to slice location. Therefore, 3D phantoms are more suitable than 2D phantoms $(7,20)$. Now, a review of different designs of $2 \mathrm{D}$ and 3D phantoms for evaluating MR images distortion is presented here.

Price et al. used low-density polyurethane foam plates and paintball polyethylene bases as markers in a 3D phantom in order to evaluate image distortion in three MR systems with different bore sizes. The researchers found that the image distortion is less than $1 \mathrm{~mm}$ in distances $<10 \mathrm{~cm}$ from the isocenter point. In contrast, distortion increased for more distances, and it varied for the 3 MR systems (21). Walker et al. used FLUKE biomedical phantom in their study and investigated the amount of MR image distortion in four clinical Siemens scanners with different imaging protocols. It was shown that an increase in BW $(7.7-76.8 \mathrm{kHz})$ can reduce maximum distortion from 6.33 to 1.74 $\mathrm{mm}$. Moreover, as BW increased, the signal to noise ratio (20.6-6.54) decreased. Price et al.'s study proved that the amount of distortion is dependent on the scanner type and the protocols used in the imaging (22). In addition, Wang et al. (2004) introduced a new 3D Perspex cubic phantom filled with mineral oil consisting of parallel plastic grids with external dimensions

Int. J. Radiat. Res., Vol. 18 No. 4, October 2020 
of $310 \mathrm{~mm} \times 310 \mathrm{~mm} \times 310 \mathrm{~mm}$ in order to evaluate MR image distortion (7). The authors used the cubic phantom to compare distortion of four MRI scanners. Their comparison showed that maximum distortion of the scanners ranges between 10 and $25 \mathrm{~mm}{ }^{(18)}$. Wang et al. provided a method to correct the geometric distortion, which can interpolate between markers and maximum distortion reached about $0.8 \mathrm{~mm}$ (23). Torfeh et al. developed a large field of view phantom containing 357 rods by $2 \mathrm{~mm}$ intervals placed in the water-filled Perspex coat with 420 $\mathrm{mm}$ in diameter. The results showed that if the vendor 3D geometric distortion correction algorithm is used, the mean distortion would be distributed between 0.21 and $0.86 \mathrm{~mm}$ in the radial distances up to $20 \mathrm{~cm}{ }^{(24)}$. Another study conducted by Torfeh et al. designed a new phantom containing light foam layers with a matrix of 2504 ellipsoidal markers to evaluate distortion. In a case of the use of the 2D correction algorithms, maximum distortion reduced from $8.52 \mathrm{~mm}$ to $2.34 \mathrm{~mm}$, while the 3D correction program reduced it to $1.95 \mathrm{~mm}$ (25). Moreover, Walker et al. extended a new 3D volumetric phantom by plastic layers containing vitamin $\mathrm{E}$ capsules. The researchers investigated both 2D and $3 \mathrm{D}$ acquisition sequences with the standard static and the moving table. Their results indicated that maximum distortion decreases with the imaging by moving table, but the blurring effect increases with the table speed; however, the most acceptable geometric accuracy was seen in table speed of $1.1 \mathrm{~mm} / \mathrm{s}$ (11).

Some researchers used 3D grid-shaped of plastic with the dimensions of $170 \mathrm{~mm} \times 170$ $\mathrm{mm} \times 250 \mathrm{~mm}$ to evaluate the image distortion of six MR scanners of Siemens and Philips companies. According to this phantom dimensions, the image distortion was less than 2 $\mathrm{mm}$ for all points (26). In addition, Mah et al. examined the effect of image distortion on the lateral and anterior-posterior (AP) separation of 12 patients via vendor distortion correction program. The contouring error caused by geometric distortion for the patient with separation less than $40 \mathrm{~cm}$ has been shown to be in an acceptable range with $1 \%$ error in dose calculation stage (27). Moreover, Damyanovich et al. designed a 7D grid phantom to analyze MR images distortion of the stereotactic radiosurgery applications and in the field of view $24 \mathrm{~cm}$. The researchers reported that the amount of image distortion is less than $2.5 \mathrm{~mm}$ (28).

Different studies showed complexity of the system related geometric distortion and its dependence on multiple factors such as the kind of the system used, pulse sequence type (gradient echo (GRE) and spin echo (SE)), imaging parameters (echo time (TE), repetition time (TR), and receiver bandwidth)), 2D or 3D sequences, and distances from the system isocenter $(3,17)$. Therefore, it is necessary to evaluate the amount of image distortion in a large field of view for each MR simulator due to the complications of the MR image distortion and its effect on the MR-based radiotherapy procedure.

The present study aimed at evaluating geometric distortion of four MRI protocols in a Siemens MR scanner through a new phantom. Therefore, the study focused on GNL. Thus, a new large field of view phantom was made, which could caver all the bore spaces of $60 \mathrm{~cm}$ and provide the grounds for attaining geometric information of the images in all slices without any gap in imaging procedure. It should be noted that the study novelties are the newly designed phantom and the use of a combination of 3D slicer and Matlab software to evaluate and quantify MR geometric distortion.

\section{MATERIALS AND METHODS}

\section{The 3D phantom}

The plastic pipes with the inner diameter of 5 mm containing water were used to build an in-house phantom in order to evaluate distortion of MR images (figure 1(A)). The phantom dimensions are $48 \times 48 \times 37 \mathrm{~cm}^{3}$ with the weight of $\sim 15 \mathrm{~kg}$, and the pipes spaced $20 \mathrm{~mm}$ apart between the nearest ones to provide a detailed mapping of the spatial distortion. This arrangement provided 325 control points for each slice. In addition, five Perspex sheets (three 
sheets with a thickness of $10 \mathrm{~mm}$ and two of them with a thickness of $2 \mathrm{~mm}$ ) were drilled to make hills for inserting the pipes. Then, the drilled slabs were stacked on a Perspex sheet as a base, and the pipes were transmitted from the hills. In the next step, one side of the pipes was closed, and the pipes were filled with water as a signal generator for CT and MR imaging. Figure 1 depicts an image of the phantom and CT image of one slice.

\section{CT imaging}

CT reference images have been shown tho be acquired at $90 \mathrm{kVp}$ and $110 \mathrm{mAs}, \sim 180$ slices, $512 \times 512$ pixel matrix with a $3-\mathrm{mm}$ thickness and zero gaps in the transverse plane for the whole phantom using a large-bore $(70 \mathrm{~cm})$ multislice CT scanner of Neusoft Company of the United States.

\section{MR imaging}

A $60 \mathrm{~cm}$ bore MAGNETOM $®$ Symphony Syngo 1.5 T from Siemens Company of Germany was used to perform MRI acquisitions. Some sequences were examined on the basis of their clinical applications for treatment planning. In order to explore the dependence of the geometric distortion on the type of sequences and image parameters, the phantom images were acquired from four different protocols (three sequences from SE and one from GE sequences) through a $2 \mathrm{D}$ vendor correction algorithm. Table 1 summarizes the details of the acquired protocols.
A

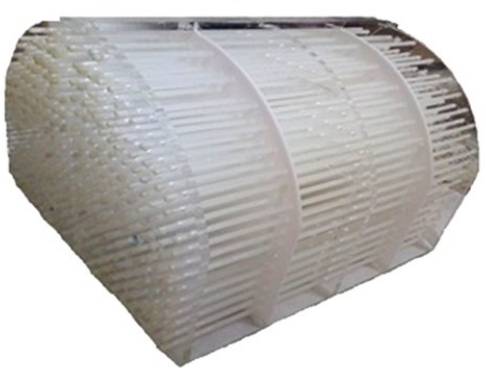

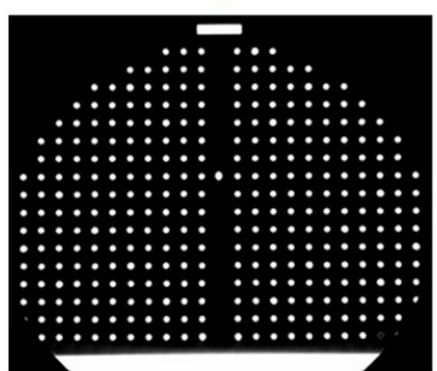

Figure 1. (A) The 3D phantom with water pipes and (B) the CT image of one slice.

Figure 1. Modified Ondo Google Satellite Map Showing Zones of Sample Collection. Map data (C2017 Google (14)

\begin{tabular}{|c|c|c|c|c|}
\hline \multirow{2}{*}{ parameters } & \multicolumn{4}{|c|}{ Protocol name } \\
\hline & t2_haste_tra_bh & t2_tse_tra & t2_tirm_fs_tra_mbh & t1_fl2d_tra_mbh \\
\hline $\begin{array}{c}\text { Properties of the } \\
\text { protocol }\end{array}$ & $\begin{array}{c}\text { Half-Fourier-Acquired } \\
\text { Single-shot Turbo spin } \\
\text { Echo (HASTE) }\end{array}$ & $\begin{array}{c}\text { Fast spin echo (FSE) } \\
\text { imaging, also known } \\
\text { as Turbo spin echo } \\
\text { (TSE) imaging }\end{array}$ & $\begin{array}{l}\text { Turbo inversion recovery } \\
\text { magnitude (TIRM), a type } \\
\text { of inversion recovery pulse } \\
\text { sequence for fat } \\
\text { suppressing signal }\end{array}$ & $\begin{array}{l}\text { Fast low angle shot } \\
\text { magnetic resonance } \\
\text { imaging (FLASH) }\end{array}$ \\
\hline Scanning Sequence & SE & SE & IR\SE & GE \\
\hline MR Acquisition Type & $2 D$ & $2 \mathrm{D}$ & $2 \mathrm{D}$ & $2 \mathrm{D}$ \\
\hline Slice Thickness & $3 \mathrm{~mm}$ & $3 \mathrm{~mm}$ & $3 \mathrm{~mm}$ & $3 \mathrm{~mm}$ \\
\hline Spaces between slices & $0 \mathrm{~mm}$ & $0 \mathrm{~mm}$ & $0 \mathrm{~mm}$ & $0 \mathrm{~mm}$ \\
\hline Repetition Time & 531 & 10000 & 10000 & 539 \\
\hline Echo Time & 78 & 98 & 99 & 4.76 \\
\hline Spacing Between Slices & $3 \mathrm{~mm}$ & $3 \mathrm{~mm}$ & $3 \mathrm{~mm}$ & $3 \mathrm{~mm}$ \\
\hline Pixel Band width & 475 & 130 & 305 & 160 \\
\hline Flip Angle & 150 & 150 & 150 & 70 \\
\hline
\end{tabular}




\section{Assessment of MR image distortion}

The Matlab (version R2014a (8.3.0.532 () program in combination with Markups module of 3D slicer software (version 4.8.1) was used to quantitatively assess the image geometric distortion. For each slice, the markers coordinate information was acquired by Markups module of 3D slicer. The center information of CT was used as a reference image. In addition, MRI markers for the slices by intervals of $1 \mathrm{~cm}$ were saved as .fcsv files and imported to Matlab. Then, an in-house program was used to provide a complete quantitative assessment of the geometric distortion for all slices of four protocols. CT images determined the coordinates and radial distances of markers for each slices. Therefore, each marker position in CT images was considered as a reference, and the position difference of MR markers (as displaced markers) and CT marker were reported as the amount of distortion. Figure 2 provides a summary of the stages needed for the evaluation and quantification of geometric distortion.

\section{Reproducibility of MR images distortion}

In order to examine the reproducibility of the geometric distortion of the MR images, the four protocols were repeated three times within a month's interval in the same position set up.

\section{RESULTS}

\section{Image distortion in different MR protocols}

The distance from the isocenter point of MR scanner for each marker was calculated as radial distance. The displacement amount of markers in MR images was plotted according to radial distance in different sequences shown in figure 3.

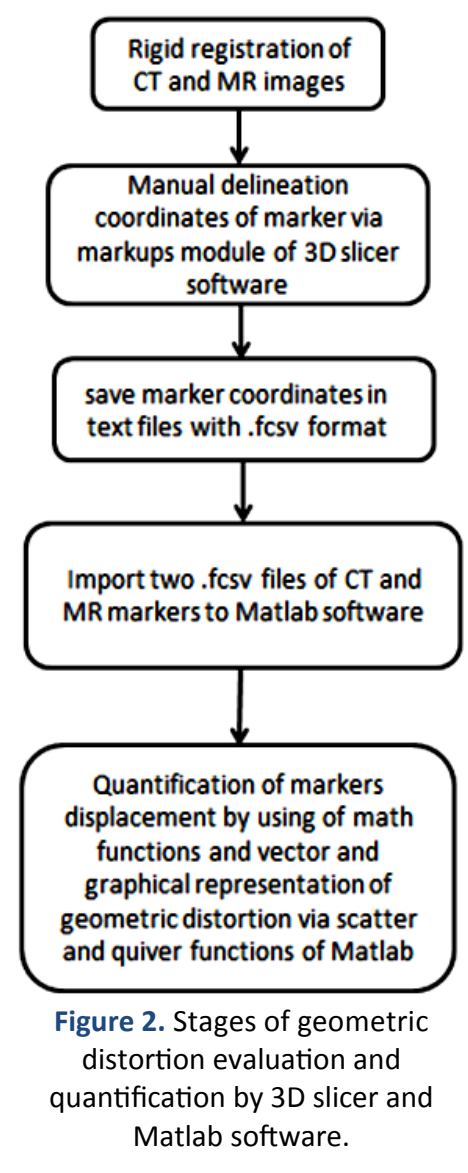

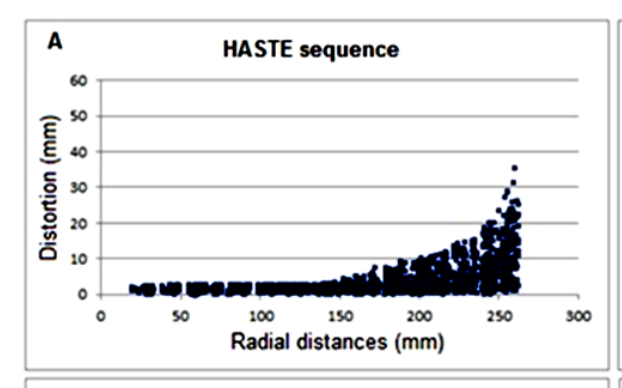
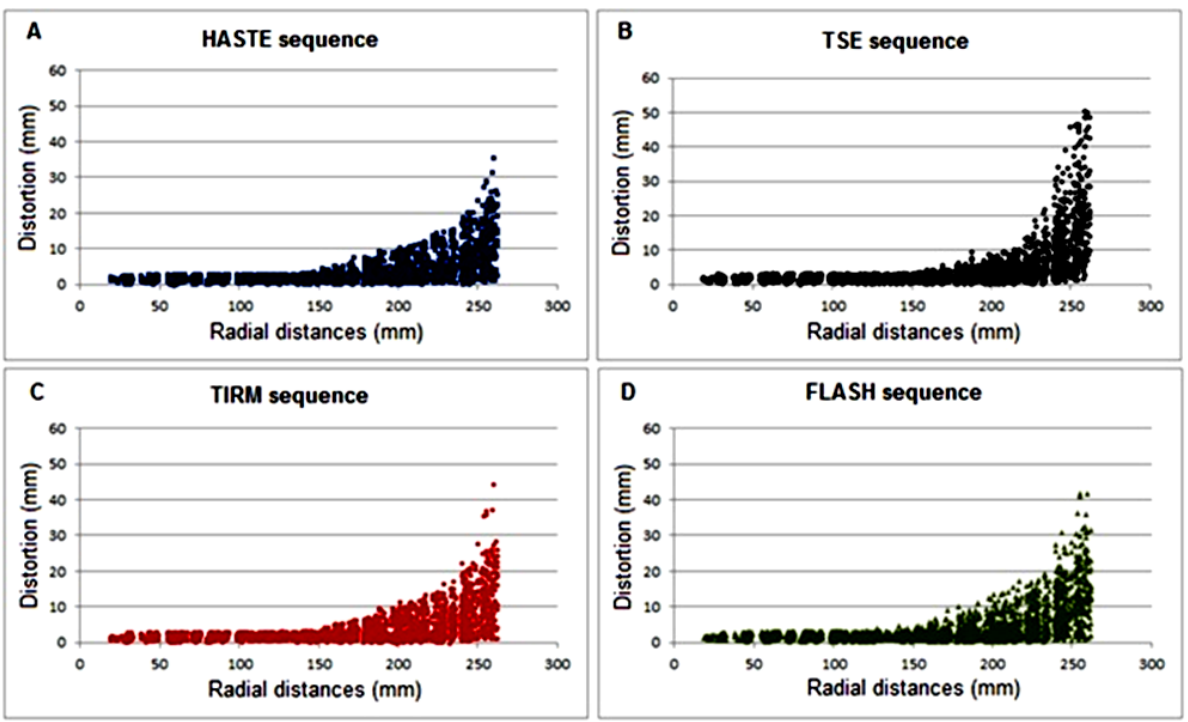

Figure 3. The displacement amount of markers in MR images according to radial distance from isocenter point in different sequences. 
The results showed that the amount of distortion for each protocol is under $2 \mathrm{~mm}$ in the radial distances $<10 \mathrm{~cm}(\mathrm{FOV}=20 \mathrm{~cm})$; however, distortion increased in radial distances $>10 \mathrm{~cm}$, and reached about $5 \mathrm{~cm}$ in radial distances greater than $25 \mathrm{~cm}$.

It should be noted that the amount of distortion is protocol-dependent for the radial distances more than $10 \mathrm{~cm}$. However, the present study revealed that the maximum distortion is related to TSE sequence with 5.054 $\mathrm{cm}$ distortion in the distance of $26.197 \mathrm{~cm}$.

Figure 4 shows a slice of the phantom in +10 $\mathrm{cm}$ location of CT and for different MR protocols. According to the results, the pattern of distortion is different in the same $\mathrm{Z}$ for different protocols.
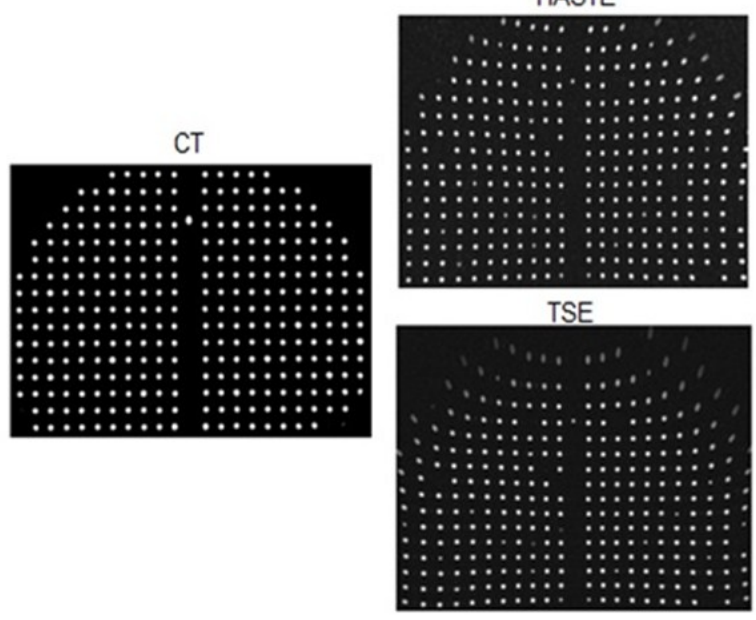

TIRM

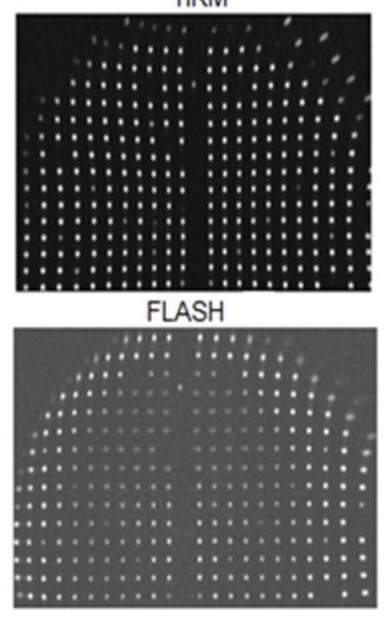

Figure 4. The slice images in $+10 \mathrm{~cm}$ location of $\mathrm{CT}$ and four MR protocols.
In particular, geometric distortion of the HASTE sequence, which is a common clinic protocol for MR imaging of the pelvic region, was investigated. Figure 5 presents the sagittal and coronal CT and MR HASTE images of the phantom. Distortion can be clearly observed in the edges of MR image in comparison with CT images. The images of coronal and sagittal view indicated dependence of distortion on the slice location in the $\mathrm{Z}$ direction.

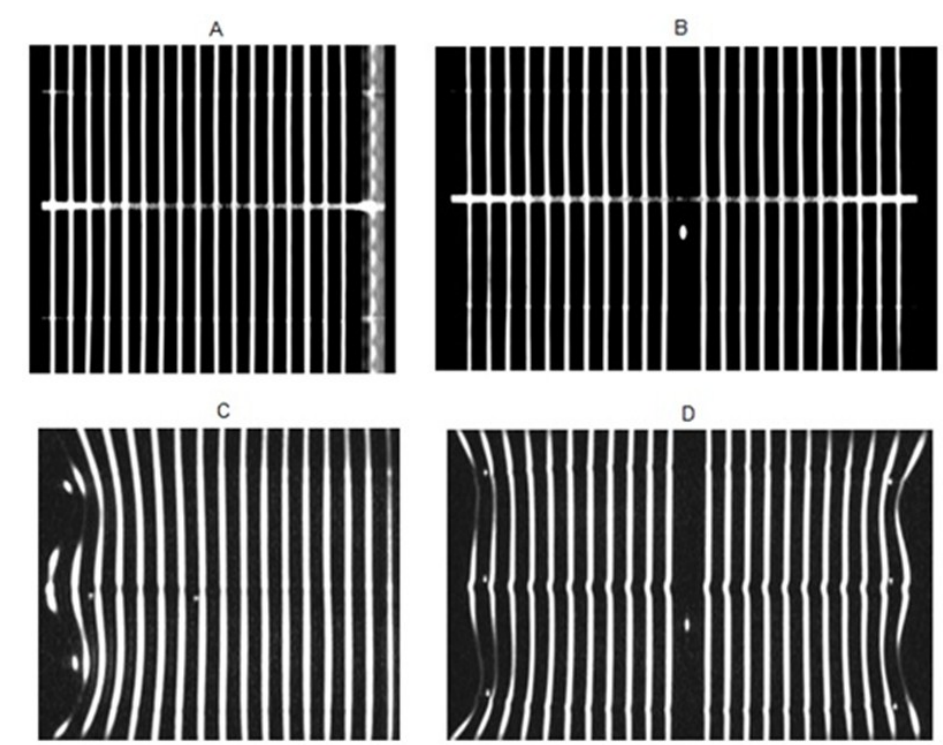

Figure 5. The sagittal and coronal CT ( $A$ and $B$ ) and MR ( HASTE sequence ( $C$ and $D)$ ) images of the phantom. 
Figure 6 depicts the displacement vector of different slices of one protocol (HASTE) in the locations of $0,5,10$ and $15 \mathrm{~cm}$ from the central slice. A vector with the length of $2 \mathrm{~cm}$ was placed in all images in order to make the best comparison.

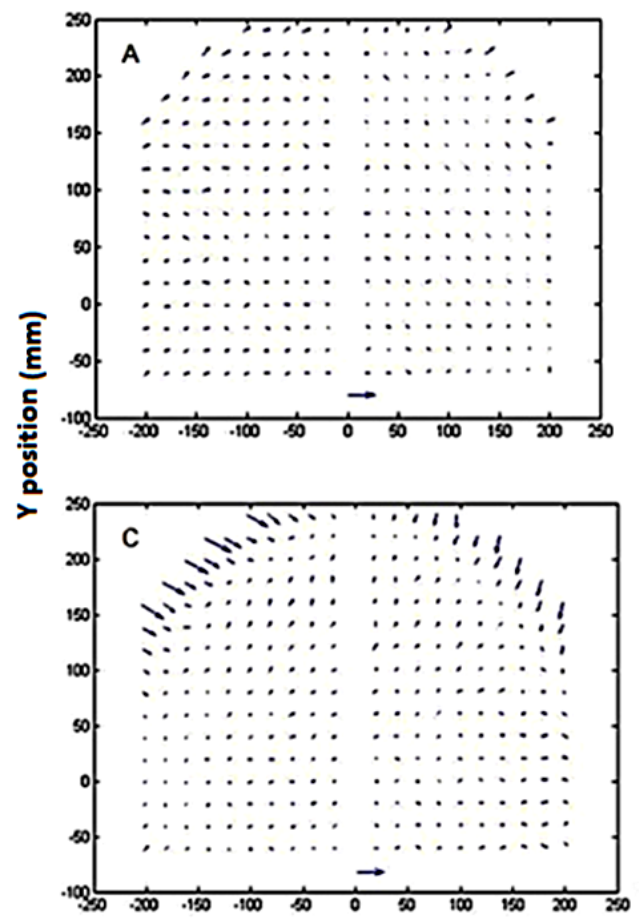

As shown by all vector figures, the pattern of image distortion differs for one protocol in different slice locations, and there is not any symmetry in the amount and the pattern of geometric distortion.
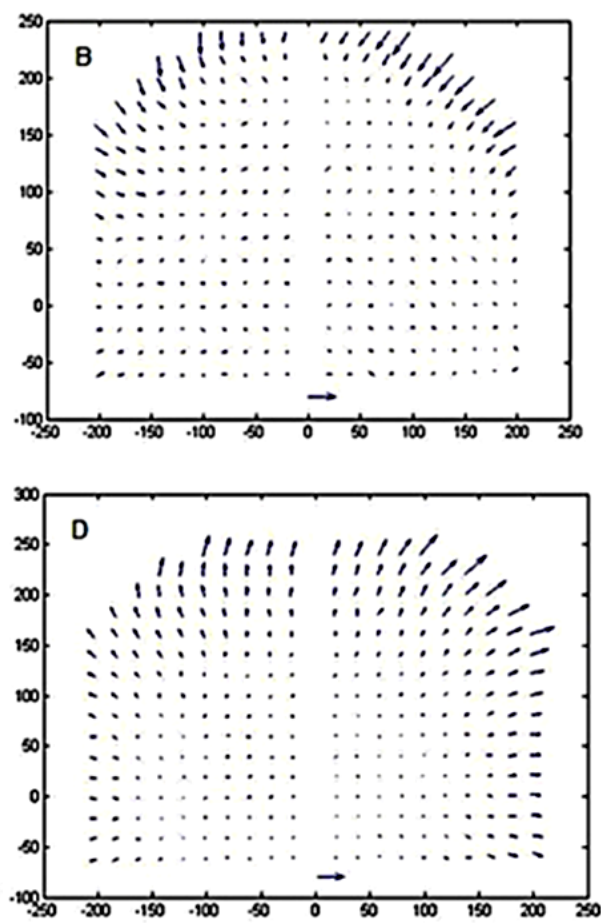

$X$ position $(\mathbf{m m})$

Figure 6. The displacement vector of different axial slices of HASTE protocol ( $A:+0 \mathrm{~cm}, B:+5 \mathrm{~cm}, \mathrm{C}:+10 \mathrm{~cm}$ and $\mathrm{D}:+15 \mathrm{~cm}$ ). Horizontal and vertical axis show $X$ and $Y$ positions in $\mathrm{mm}$, respectively.

\section{Reproducibility test}

MR imaging of four protocols from the phantom in the same position was implemented three times in month intervals in order to evaluate the reproducibility of geometric distortion. The results obtained from the imaging showed that differences of markers location due to geometric distortion are less than $1 \mathrm{~mm}$ for all protocols that is an acceptable value (28). Therefore, it could be stated that geometric distortion is stable for all protocols in three months.

\section{DISCUSSION}

For MR-based planning, it is essential to evaluate geometric distortion evaluation for image protocols before the correction of patient positioning and conversion of MR pixel values into Hounsfield units. Another study designed different 2D and 3D phantoms in order to investigate the geometric distortion of $\mathrm{MR}$ images. The present study used a 3D large field of view phantom composed of 325 plastic water pipes for assessing the geometric distortion of four MR protocols (HASTE, TSE, TIRM, and FLASH) through 2D vendor correction algorithm of the system. The large field of the view phantom and the use of pipes instead of the marker allowed us to assess the distortion slice by slice with thin slice thickness and without any gap to increase precision in the evaluation of geometric distortion.

Comparison of the total distortion according to the radial distances from the isocenter point (figure 3) showed that the distortion is acceptable for radiotherapy planning in FOVs $<20 \mathrm{~cm}$; however, it increased rapidly for more 
radial distance, which should be corrected for radiotherapy applications. The data for the amount of distortion according to radial distances agree with other studies $(7,16,21,22,27$, 28).

As seen in the diagrams plotted in figures 3 and 4, the amount and pattern of distortion is dependent on the type of protocol. In addition, for different anatomical locations, special MR protocols have been shown to be suitable for accurate diagnostic and good resolution and SNR. As a result of the dependence of distortion on the protocol, it is necessary to initially choose a proper protocol for MR-based planning according to the anatomical location. Then, distortion should be evaluated and corrected for MR planning before the contouring stage. One of the studies about the head region with the FOV $<20 \mathrm{~cm}$ showed that the amount of distortion is an acceptable range for radiotherapy planning, which has been confirmed by the results of the present study. However, for the anatomical locations with large FOV such as chest, abdomen, and pelvic regions, an appropriate correction should be implemented before planning (22).

For the protocols investigated in the present study, geometric distortion has been reported to be higher for the TSE sequence. Comparison of the protocols BW showed that the BW of TSE is less than the others. In contrast, several studies showed that the increased BW is one of the critical factors for reducing both system-specific and susceptibility-induced distortions $(22,29,30)$. Interestingly, multiple studies suggested the BW at least $440 \mathrm{~Hz} /$ pixel for MR-based planning; however, as BW increased, the SNR decreased. Therefore, it would be necessary to be a trade-off between SNR and geometric fidelity (11). It should be noted that the HASTE sequence with the BW of $475 \mathrm{~Hz} /$ pixel has the lowest distortion, and the TSE sequence with the BW of $130 \mathrm{~Hz} /$ pixel has the most distortion, which is in agreement with the similar studies conducted. Thus, these results confirm the importance of the BW for MR imaging with radiotherapy purposes.

For each protocol, the amount and pattern of distortion were not the same in all slices. Put differently, it depended on the slice location for each imaging protocol (figures 5 and 6). Therefore, a proper protocol for MR-based radiotherapy should be evaluated and corrected slice by slice with regard to the dependence of the geometric distortion on the type of protocol and slice location.

Designing a phantom with water pipes could provide an accurate examination of geometric distortion in $\mathrm{X}$ and $\mathrm{Y}$ directions for all slices without any interpolations; however, it could not detect a shift in signal intensity along the $z$-axis, which is one of the limitations of our study. On the other hand, the amount of distortion in $\mathrm{X}$ and $\mathrm{Y}$ directions were highly taken into account in this study because they can have the greatest effect in external contour determination for MR only planning in the dosimetry calculation stage through synthetic CT images. In the contouring stage of internal organs, the MR images can be acquired in the small field of views with a little geometric distortion. In addition, the $\mathrm{X}$ and $\mathrm{Y}$ geometric distortion in the large field of view images for the external contour has the greatest effect on the anterior-posterior and lateral separation, which are important during the stage of dosimetry calculations.

The present study aimed to evaluate the system geometric distortion of four MR protocols through a new three-dimensional (3D) large field of view phantom. Moreover, the use of CT and MR images of the phantom as well as point and vector diagram of geometric distortion showed that geometric distortion for each MR scanner is dependent on the RF sequence pulse (SE or GE) and image parameter (TE, TR, and receiver bandwidth). The geometric distortion can be ignored for the $\mathrm{FOV}<20 \mathrm{~cm}$ (for the head region) and should be evaluated and corrected for more FOVs before the MR only radiotherapy.

\section{CONCLUSION}

The designed 3D large field of view phantom and the method presented in this study can provide sufficient information about the system 
related to geometric distortion. As the results of the study showed, the use of the 2D vendor correction program could reduce the geometric distortion to an acceptable range of FOVs $<20$ $\mathrm{cm}$. However, for more FOVs, it is crucial to use further correction for MR treatment planning purposes. This study quantitatively investigated the geometric distortion. It should be noted that our future research will focus on the correction of the HASTE MR image for the MR planning of the pelvic region.

\section{ACKNOWLEDGEMENTS}

This paper was extracted from a PhD thesis of Medical Physics. The authors would like to thank the Research Deputy of MUMS for financial support of this project, numbered (961527).

\section{Conflicts of interest: Declared none.}

\section{REFERENCES}

1. Dowling JA, Lambert J, Parker J, Salvado O, Fripp J, Capp A (2012) An atlas-based electron density mapping method for magnetic resonance imaging (MRI)-alone treatment planning and adaptive MRI-based prostate radiation therapy. Int J Radiat Oncol Biol Phys, 83(1): 5-11.

2. Burgos N, Guerreiro F, McClelland J, Presles B, Modat M, Nill $S$ (2017) Iterative framework for the joint segmentation and CT synthesis of MR images: application to MRIonly radiotherapy treatment planning. Phys Med Biol, 62 (11): 4237-4253.

3. Prabhakar R, Julka P, Ganesh T, Munshi A, Joshi R, Rath G (2007) Feasibility of using MRI alone for 3D radiation treatment planning in brain tumors. Jpn J Clin Oncol, 37(6): 405 $-411$.

4. Tyagi N, Fontenla S, Zhang J, Cloutier M, Kadbi M, Mechalakos J (2017) Dosimetric and workflow evaluation of first commercial synthetic CT software for clinical use in pelvis. Phys Med Biol, 62(8): 2961-2975.

5. Dai $Y$ and King A (2018) State of the art MRI in head and neck cancer. Clin Radiol,73(1): 45-59.

6. Yu H, Caldwell C, Balogh J, Mah K (2014) Toward magnetic resonance-only simulation: segmentation of bone in MR for radiation therapy verification of the head. I Int I Radiat Oncol Biol Phys, 89(3): 649-657.

7. Wang D, Doddrell DM, Cowin G (2004) A novel phantom and method for comprehensive 3-dimensional measure- ment and correction of geometric distortion in magnetic resonance imaging. Magn Reson Imaging, 22(4): 529-542.

8. Menzel HG (2014) International Commission on Radiation Units and Measurements. Journal of the ICRU, 14(2): 1-2.

9. Ahnesjö A and Aspradakis MM (1999) Dose calculations for external photon beams in radiotherapy. Phys Med Biol, 44(11): R99-155.

10. Korhonen J, Kapanen $M$, Keyriläinen J, Seppälä $T$, Tenhunen M (2014) A dual model HU conversion from MRI intensity values within and outside of bone segment for MRI-based radiotherapy treatment planning of prostate cancer. Med Phys, 41(1): 011704.

11. Walker A, Liney G, Holloway L, Dowling J, Rivest-Henault D, Metcalfe P (2015) Continuous table acquisition MRI for radiotherapy treatment planning: distortion assessment with a new extended 3D volumetric phantom. Med Phys, 42(4):1982-1991.

12. Fransson A, Andreo P, Pötter R (2001) Aspects of MR image distortions in radiotherapy treatment planning. Strahlenther Onkol, 177(2): 59-73.

13. Schmidt MA and Payne GS (2015) Radiotherapy planning using MRI. Phys Med Biol, 60(22): R323-61.

14. Stanescu T, Hans-Sonke J, Stavrev P, Fallone BG (2006) ЗT MR-based treatment planning for radiotherapy of brain lesions. Radio Oncol, 40(2): 125-132.

15. Jonsson JH, Karlsson MG, Karlsson M, Nyholm T (2010) Treatment planning using MRI data: an analysis of the dose calculation accuracy for different treatment regions. Radiat Oncol, 5: 62.

16. Price RG, Kadbi M, Kim J, Balter J, Chetty IJ, Glide-Hurst CK (2015) Characterization and correction of gradient nonlinearity induced distortion on a $1.0 \mathrm{~T}$ open bore MR-SIM. Med Phys, 42(10): 5955-5960.

17. Weygand J, Fuller CD, Ibbott GS, Mohamed AS, Ding Y, Yang J, et al. (2016) Spatial precision in magnetic resonance imaging-guided radiation therapy: the role of geometric distortion. Int J Radiat Oncol Biol Phys, 95(4): 13041316.

18. Wang D, Strugnell W, Cowin G, Doddrell DM, Slaughter R (2004) Geometric distortion in clinical MRI systems: Part I: evaluation using a 3D phantom. Magn Reson Imaging, 22 (9): 1211-1221.

19. Mallozzi R (2015) Geometric distortion in MRI. The Phantom Laboratory, Inc; 2015.

20. Wang D and Doddrell DM (2005) Geometric distortion in structural magnetic resonance imaging. Current Medical Imaging Reviews, 1(1): 49-60.

21. Price RG, Knight RA, Hwang KP, Bayram E, Nejad-Davarani SP, Glide-Hurst CK (2017) Optimization of a novel large field of view distortion phantom for MR-only treatment planning. J App/ Clin Med Phys, 18(4): 51-61.

22. Walker A, Liney G, Metcalfe P, Holloway L (2014) MRI distortion: considerations for MRI based radiotherapy treatment planning. Australas Phys Eng Sci Med, 37(1): 103-113.

23. Deming Wang, Wendy Strugnell, Gary Cowin, David M. Doddrell, Richard Slaughter (2004) Geometric distortion in 
clinical MRI systems Part II: correction using a 3D phantom. Magn Reson Imaging, 22(9): 1223-1232.

24. Torfeh T, Hammoud R, McGarry M, Al-Hammadi N, Perkins G (2015) Development and validation of a novel large field of view phantom and a software module for the quality assurance of geometric distortion in magnetic resonance imaging. Magn Reson imaging, 33(7): 939-949.

25. Torfeh T, Hammoud R, Perkins G, McGarry M, Aouadi S, Celik A (2016) Characterization of 3D geometric distortion of magnetic resonance imaging scanners commissioned for radiation therapy planning. Magn Reson Imaging, 34 (5): 645-653.

26. Jafar M, Jafar YM, Dean C, Miquel ME (2017) Assessment of Geometric Distortion in Six Clinical Scanners Using a 3DPrinted Grid Phantom. J Imaging, 3(3): 28.

27. Mah D, Steckner M, Hanlon A, Freedman G, Milestone B, Mitra R (2002) MRI simulation: effect of gradient distor- tions on three-dimensional prostate cancer plans. Int $J$ Radiat Oncol Biol Phys, 53(3): 757-765.

28. Damyanovich A, Rieker M, Zhang B, Bissonnette J, Jaffray $D$ (2018) Design and implementation of a 3D-MR/CT geometric image distortion phantom/analysis system for stereotactic radiosurgery. Phys Med Biol, 63(7):075010.

29. Lundman JA, Bylund M, Garpebring A, Karlsson CT, Nyholm T (2017) Patient-induced susceptibility effects simulation in magnetic resonance imaging. Phys Imaging Radiat Oncol, 1: 41-45.

30. Adjeiwaah M, Bylund M, Lundman JA, Karlsson CT, Jonsson JH, Nyholm T (2018) Quantifying the Effect of 3T Magnetic Resonance Imaging Residual System Distortions and Patient-Induced Susceptibility Distortions on Radiation Therapy Treatment Planning for Prostate Cancer. Int J Radiat Oncol Biol Phys, 100(2): 317-324. 